\title{
La carga del cuidado en la enfermedad crónica en la díada cuidador familiar-receptor del cuidado'
}

\author{
Beatriz Sánchez Herrera² \\ Lorena Chaparro Díaz³ \\ Gloria Mabel Carrillo González4
}

doi:10.11144/Javeriana.ie18-2.ccec

Cómo citar: Sánchez Herrera B, Chaparro Díaz L, Carrillo González GM. La carga del cuidado en la enfermedad crónica en la diada cuidador familiar-receptor del cuidado. Investig Enferm. Imagen Desarr. 2016;18(2): 43-60. doi:10.11144/Javeriana.ie18-2.ccec

1. Artículo derivado de investigación. Fecha de recepción: 20 de marzo de 2015. Fecha de aceptación: 4 de diciembre de 2015.

2. Master in Science of Nursing. Profesora titular de la Facultad de Enfermeria, Universidad Nacional de Colombia. Correo electrónico: cbsanchezh@unal.edu.co

3. Doctora en Enfermería. Profesora asociada de la Facultad de Enfermería, Universidad Nacional de Colombia. Correo electrónico: olchaparrod@unal.edu.co

4. Magíster en Enfermería. Profesora asociada de la Facultad de Enfermeria, Universidad Nacional de Colombia. Correo electrónico: gmcarrillog@unal.edu.co 


\section{Resumen}

Objetivo: Analizar cómo se aborda y se mide la carga del cuidado en la enfermedad crónica no transmisible (ECNT) en la díada compuesta por el cuidador familiar y el receptor del cuidado. Método: Revisión integrativa de literatura en las bases de datos CINAHL, Ovid, SciELO, Medline y PsycInfo. Los estudios se clasificaron y analizaron según la conceptualización de la díada cuidador familiar-receptor del cuidado, su interacción, sus componentes y sus indicadores de medición. Resultados: $\mathrm{El}$ análisis establece seis categorias de instrumentos: 1) las que consideran al grupo familiar como contexto de la díada, 2) las que ven a las personas con enfermedad crónica como integrantes de la díada, 3) las que ven a los cuidadores familiares como integrantes e informantes de la díada, 4) las que analizan la perspectiva de los pacientes y la de su grupo familiar como una mirada interna y externa de la díada, 5) las que toman la díada propiamente dicha y 6) las que analizan la perspectiva del cuidador externo a la díada. Conclusión: Es ampliamente aceptado que el fenómeno de la carga de la ECNT afecta a la díada cuidador familiar-receptor del cuidado; pero el enfoque con el que se reconoce modifica de manera importante su valoración. Comprender esta diferencia es necesario para elaborar propuestas de disminución de la carga del cuidado de la enfermedad crónica que a su vez mejoren el bienestar de las díadas.

Palabras clave: costo del cuidado; cuidadores; enfermedad crónica; pacientes; relaciones familiares; cuestionarios

\section{The responsibility of caregiving in chronic disease in the dyad family carer - care recipient}

\section{Abstract}

Objective: To analyze how the responsibility of caregiving for chronic non-communicable disease (NCD) is addressed and measured in the dyad comprised by the family caregiver and care receiver. Method: Integrative review of literature on the data base CINAHL, Ovid, SciELO, Medline and PsycINFO. Studies were classified and analyzed according to the conceptualization of the dyad family carer - care recipient, their interaction, their components and measurement indicators. Results: The analysis establishes six categories of instruments: (1) the ones that consider the family as the context of the dyad, (2) the ones that see people with chronic disease as members of the dyad, (3) those who see family caregivers as members and informers of the dyad, (4) those who analyze the perspective of patients and their family as an internal and external view of the dyad, (5) the ones that take the dyad itself, and (6) those who analyze the perspective of the external- to- the dyad caregiver. Conclusion: It is widely accepted that the phenomenon of the responsibility of NCDs affect the dyad family caregivercare receiver; But the approach with which it is recognized significantly modifies its valuation. Understanding this difference is necessary to develop proposals for reducing the burden of chronic disease care which in turn improve the welfare of dyads.

Keywords: care costs; caregivers; chronic disease; patients; family relationships; questionnaires 


\section{A carga do cuidado em doenças crônicas na diade cuidador familiar-receptor do cuidado}

\section{Resumo}

Objetivo: Analisar como é abordada e medida a carga do cuidado nas doenças crônicas não transmissiveis (DCNT) na díade composta pelo cuidador familiar e o receptor do cuidado. Método: Revista integrativa de literatura nas bases de dados CINAHL, Ovid, SciELO, Medline e PsycInfo. Os estudos foram classificados e analisados segundo a conceituação da díade cuidado familiar-receptor do cuidado, sua interação, componentes e indicadores de aferição. Resultados: A análise estabelece seis categorias de instrumentos: 1) das pessoas que acham o grupo familiar como contexto da díade, 2) das que veem o pessoal com doença crônica como integrantes da diade, 3) das que veem os cuidadores familiares como integrantes e informantes da diade, 4) as que analisam a perspectiva dos pacientes e do seu grupo familiar como um olhar interno e externo da díade, 5) as que pegam a diade propriamente tal e 6) as que analisam a perspectiva do cuidador externo à díade. Conclusão: É amplamente aceito que o fenómeno da carga da DCNT afeta à díade cuidador familiar-receptor do cuidado; mas o enfoque com que se reconhece modifica grandemente a sua valoração. Compreender esta diferença é preciso para elaborar propostas de diminuição da carga do cuidado da doença crônica que, por sua vez, melhorem o bem-estar das díades.

Palavras-chave: custo do cuidado; cuidadores; doença crônica; pacientes; relações familiares; inquéritos 


\section{Introducción}

En la actualidad las personas con enfermedades crónicas no transmisibles (ECNT) y sus familias asumen responsabilidades del cuidado en coparticipación, negociación y con procesos de adaptación rápidos y permanentes ante los procesos de la enfermedad $(1,2)$. En estas relaciones de cuidado y dependencia se presenta la conformación de las diadas cuidador familiar y receptor del cuidado (D CF-R), que surgen como una opción del desarrollo humano, en las que se requieren dos personas o, en el caso del autocuidado, un diálogo interno con el propio ser.

Chaparro describió el proceso de conformación de la DCF-R como sujeto de cuidado en las situaciones de ECNT y señala que este inicia con la situación de dependencia funcional, permanece en el tiempo y genera acciones mutuas a partir de un contrato de elementos personales de sus integrantes (3). La DCF-R se consolida cuando hay mutualidad y reciprocidad (4). Chaparro afirma que el vínculo especial de cuidado que une la D CF-R inicia su construcción con la manifestación de los síntomas de la enfermedad, en medio de las restricciones y con un alto grado de vulnerabilidad social, laboral y económica. La DCF-R tiene una relación íntima, por lo que se empieza a reconocer y expresar como un sujeto con identidad propia. Las fases del proceso pasan por vivir la limitación y la necesidad de ayuda, el reto o compromiso hasta llegar al logro y la trascendencia a un vínculo "especial" (4-6).

Por otra parte, está la relación entre la D CF-R y la carga del cuidado. Carga significa trabajo, dificultad, peso, desgaste, energía necesaria para algo. Cuando se habla de la carga de la ECNT o de la carga del cuidado de la ECNT para la D CF-R, se hace referencia al esfuerzo adicional que implica para ellos satisfacer las necesidades de cuidado de cada uno y las de ambos de forma simultánea. Este abordaje debe ser descrito y cuantificado al igual que los factores contextuales que lo aumentan o disminuyen, para poder ser atendido. Así es como la descripción de la conformación de la DCF-R como sujeto de cuidado, de su interacción y sus componentes, en relación con la carga del cuidado, es fundamental para abordar de manera adecuada la salud en las situaciones de ECNT.

La relación entre la carga del cuidado en la ECNT y el proceso de conformación de una díada sucede en tres etapas: limitación y necesidad de ayuda, el paso del reto (o compromiso) al logro y la forma de trascender a un "vínculo especial". Cada etapa se caracteriza por representar un sujeto de cuidado específico: persona con ECNT, cuidador familiar y díada, respectivamente. De igual forma, cada una tiene elementos asociados que contribuyen a la carga.

De acuerdo con lo descrito, el cuidado en la ECNT debe contemplar la díada como un sujeto de atención en salud, pasar de un paradigma individualista a uno integral, donde sean reconocidas y fortalecidas las potencialidades de cada uno, mitigadas las dificultades manifestadas en la carga y valorados por su experiencia en la forma de vivir el proceso de ECNT y no solo por la enfermedad. Los modelos actuales del cuidado crónico hacen 
un llamado a la descripción explícita de la realidad vivida por los actores del cuidado, entre los cuales se destaca la necesidad de valorar la carga del cuidado como elemento básico para mejorar las condiciones de salud y la calidad de vida.

Al comprender la necesidad de estudiar y fortalecer las DCF-R como componente estratégico de la atención integral en salud para las personas con ECNT, el presente estudio tuvo como objetivo analizar la forma en que se aborda y mide la carga del cuidado en la ECNT en las diadas compuestas por el cuidador familiar y el receptor del cuidado. Con base en esta información, se espera avanzar en la construcción de un programa para disminuir la carga de la enfermedad crónica en Colombia, que responda a las necesidades del contexto y permita incrementar el bienestar de las DCF-R que viven situaciones de cronicidad.

\section{Método}

Revisión integrativa a partir de una búsqueda exhaustiva apoyada con la herramienta bibliométrica del Metabuscador del Sistema de Bibliotecas de la Universidad Nacional de Colombia (Sinab), que abarcó el periodo 19772014, con los siguientes pasos:

1. Elaboración de la pregunta orientadora de la revisión: ¿cómo se aborda y mide la carga del cuidado en la ECNT en las DCF-R?

2. Búsqueda de literatura: en las bases de datos CINAHL, Ovid, SciELO, Medline y PsycInfo, bajo los descriptores diada, cuidador familiar, carga, costo de la enfermedad y enfermedad crónica, cruzándolas con los descriptores valoración, medición y evaluación, con su traducción al inglés. Como criterios de inclusión se consideraron artículos resultado de investigación con abordajes cuantitativos, cualitativos o mixtos; revisiones sistemáticas relacionadas; en idioma inglés o español. Como criterios de exclusión se tuvieron los artículos correspondientes a editoriales o a reflexiones sobre la temática en cuestión.

3. Recolección y análisis crítico de información: se encontraron 79 piezas de investigación, de las cuales se seleccionaron 54 reportadas en el mundo, por responder a los criterios de inclusión, es decir, abordan la DCF-R directa o indirectamente en la medición de la carga del cuidado de la ECNT. Estas se analizaron mediante una matriz generada para este propósito y que incluyó la procedencia del documento, los objetivos, la metodología, la forma de abordar la $\mathrm{DCF}-\mathrm{R}$, los resultados y las conclusiones. Adicionalmente, se tuvieron en cuenta criterios de validez, fiabilidad y aplicabilidad de las piezas seleccionadas.

Por la característica de la investigación, al ser de tipo documental, se atendieron las consideraciones éticas internacionales y de la Facultad de Enfermería de la Universidad Nacional de Colombia, haciendo hincapié en principios como la veracidad, fidelidad, no maleficiencia, beneficiencia y justicia. 


\section{Resultados}

El abordaje de la DCF-R es determinante para la comprensión de la carga del cuidado de la ECNT. Esta carga afecta al grupo familiar en conjunto; pero también a cada uno de sus integrantes. Las diferentes mediciones incluyen aspectos como la situación financiera; la interacción y relaciones sociales; los factores de estrés, en especial para el cuidador principal; el clima o funcionalidad familiar; las consecuencias negativas del dar cuidado para el receptor, cuidador y para la familia, y los aspectos de crecimiento o dominio personal y de la situación.

El análisis reportó seis formas diferentes de abordar la DCF-R en la medición de la carga del cuidado en situaciones de ECNT, así: 1) desde el grupo familiar, 2) desde el receptor del cuidado, 3) desde el cuidador familiar, 4) desde la perspectiva de los receptores como parte del grupo familiar, 5) desde la carga en la D-CF-R propiamente dicha y 6) desde un cuidador externo al grupo familiar. En la tabla 1 se presenta la distribución de los escalas dentro de estos grupos.

Tabla 1. Distribución de escalas de medición de la carga de la ECNT según la forma en que consideran la DCF-R

\begin{tabular}{|l|c|c|}
\hline \multicolumn{1}{|c|}{ Enfoque de las escalas de medición } & Núm. escalas & Porcentaje \\
\hline Desde el grupo familiar & 7 & 12,96 \\
\hline Desde el receptor del cuidado & 1 & 1,85 \\
\hline Desde el cuidador familiar & 41 & 75,92 \\
\hline $\begin{array}{l}\text { Desde la perspectiva de los receptores como } \\
\text { parte del grupo familiar }\end{array}$ & 1 & 1,85 \\
\hline Desde la carga en la D-CF-R propiamente dicha & 3 & 5,55 \\
\hline Desde un cuidador externo al grupo familiar & 1 & 1,85 \\
\hline Total & $\mathbf{5 4}$ & $\mathbf{1 0 0 , 0 0}$ \\
\hline
\end{tabular}

Fuente: elaboración propia, 2014

A partir de estos hallazgos se generó un mapa mental que refleja la compleja forma en que la DCF-R es concebida y valorada para comprender el fenómeno de la carga del cuidado en la ECNT (figura 1). 


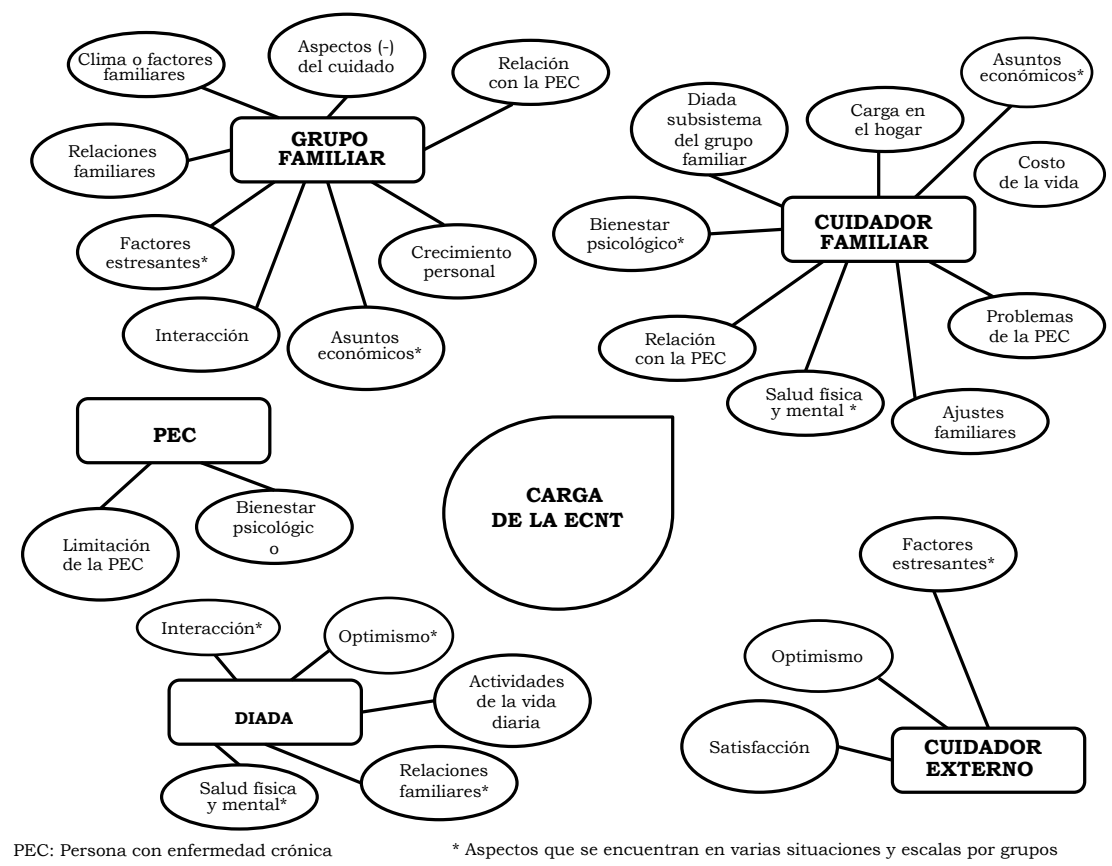

Figura 1. La D CF-R frente a la valoración de la carga de la ECNT

Fuente: elaboración propia con base en la revisión.

\section{Discusión}

\section{La DCF-R dentro del contexto del grupo familiar}

Las DCF-R dentro del contexto familiar o como un subsistema del sistema familiar se ha explorado a través de las siguientes escalas: 1) la Escala de Impacto en la Familia, empleada en familias de niños $(7,8)$. 2) La Herramienta de Valoración familiar, basada en el Modelo de Funcionalidad Familiar de McMaster (9,10). 3) La Escala del Impacto Social en el Cuidador, de Poulshock y Deimling (11), aplicada en familias que cuidan adultos mayores. 4) El Índice de Resistencia Familiar, desarrollado por McCubbin y cols., y aplicado en familias de personas en quimioterapia para describir dificultades asociadas al cuidado familiar y predecir el ánimo de los cuidadores (12). Este índice también ha sido predictor para el cuidado de enfermería de familias de niños que viven con ECNT (13). 5) Escala del Ambiente Familiar, desarrollada por Moos, y empleada en familias con cuidadores de personas con ECNT para señalar tanto los problemas como las fortalezas (14). 6) El Índice del Dar Cuidado, diseñado por Bass y Bowman, para cuidadores de personas dependientes o con ECNT, que permite describir las consecuencias negativas al cuidar (15). Y 7) Inventario de Consecuencias de Dar Cuidado Familiar, 
de Shyu y cols., desarrollada en Taiwán aplicada a familias en el rol de cuidadores y empleada por enfermería en hogares de cuidado (16).

\section{La DCF-R desde el receptor del cuidado}

En este grupo se encontró la Escala de Carga, diseñada por Simmons para abordar la autopercepción de carga de los pacientes con cáncer frente a los cuidadores (17). Llama la atención el hecho de tener un solo estudio de medición de la carga que se dirija a los pacientes, ya que son ellos quienes viven directamente la situación de la ECNT. Este estudio acepta que la carga de la ECNT se asocia con el cuidado e implica, al menos, al otro integrante de la DCF-R o al grupo familiar de personas con cáncer. Vale la pena señalar que la Escala muestra debilidades en las dimensiones de bienestar psicosocial y calidad de vida (18).

\section{La DCF-R desde el cuidador familiar}

Es de resaltar que el mayor número de escalas o instrumentos de valoración están diseñados para abordar el fenómeno de la carga del cuidado en el cuidador, dentro de las que se incluyen:

1. La Entrevista de Carga de Zarit, desarrollada por Steven Zarit para medir la carga en cuidadores de personas ancianas con demencia, y cuenta con varias versiones modificadas y conocidas como Entrevista de Carga y Entrevista Abreviada de Carga de Zarit, con pruebas psicométricas en diferentes idiomas y culturas. Está disponible en francés, japonés, chino, hebreo, coreano, español y portugués (19-22).

2. La Lista de Chequeo de las Formas de Afrontamiento de Folkman y Lazarus, aplicada a cuidadores de personas con Alzheimer (23), que tiene una versión corta propuesta por Vitaliano y cols. (24). Con base en esta se comparó la respuesta de los géneros frente a retos de cuidado (25).

3. La Escala de Estrés de los Parientes, empleada con cuidadores de personas ancianas con demencia que vivian en la comunidad y asistían a un hospital de día junto con los receptores del cuidado (26).

4. La Lista de Chequeo de Problemas de Memoria y Comportamiento, de Zarit y cols. Esta debe ser aplicada por terapeutas con los cuidadores para valorar su reacción frente al cuidado. Se recomienda el uso de la versión revisada para valoración clínica y empírica de problemas comportamentales en los pacientes con demencia. Disponible en español, mandarín, chino, alemán y japonés $(27,28)$.

5. La Versión revisada de la RMBPC.

6. La Escala de Estrés Percibido, desarrollada en cuidadores de pacientes con trauma medular y Alzheimer (susceptible de aplicar en población en general), que valora a los cuidadores a partir del último mes de la experiencia. Esta cuenta con una versión corta para aplicar por vía telefónica (30) y una versión revisada de Cole (31). 
7. El Índice de la Tensión con el Cuidado, de Robinson, para cuidadores de pacientes cardiacos de 65 y más años, sometidos en un término de 2 meses anteriores a cirugía. Esta es útil en ambientes hospitalarios y reúne la mayoría de los elementos de carga que hay en la literatura (32).

8. Las escalas de Carga Objetiva y Subjetiva de Montgomery y cols., diseñada para estudiar a cuidadores familiares de ancianos dependientes, y elaborada a partir de la experiencia de ser cuidador (33).

9. El Índice del Costo del Cuidado, de Kosberg y Cairl, aplicada en cuidadores familiares de ancianos. El estudio demostró que el índice es confiable y útil para valorar la familia, orientar la intervención y como guía en la interacción grupal de pares (34).

10. El Perfil de Estrés de Derogatis, basado en el modelo de Lazaurus y Folkman (35), empleado en cuidadores de niños con VIH positivo en fase sida y de niños con cáncer (36).

11. La Escala de Culpa, propuesta por Wells y Jorm para cuidadores de personas enfermas (37). Fue empleada posteriormente en cuidadores de personas con demencia y con discapacidad mental (38).

12. El Inventario de la Carga del Cuidado, de Novak y Guest, diseñada para estudiar cuidadores de personas ancianas con problemas cognitivos que puede ser autoadministrada y empleada en la comunidad. Permite reconocer que la dependencia de tiempo se asocia con la disfunción del paciente y con el compromiso frente al cuidado (39). Esta cuenta con la versión modificada por Caserta y cols. (40) y la versión china de Chou y cols. (41).

13. La Escala de la Estimación del Cuidado, de Lawton y cols. (42), que tiene una versión modificada de Hughes y Caliandro (43) y otra de Servick y cols. (44). Se ha empleado en cuidadores de niños con sindrome de inmunodeficiencia adquirida en fase sida, cuidadores de pacientes dependientes de ventilador en el hogar y cuidadores de adultos con trauma cerebral (45). Se recomienda su empleo, pero se sugiere revisar la que incluye una dimensión adicional.

14. La Escala del Peso al Cuidador, de Oberst y cols. Desarrollada en cuidadores de personas con cáncer que recibían quimioterapia y que permite ser autoadministrada (46).

15. La Escala de Estimación de Dar Cuidado, de Oberst y cols. (46). Aplicada a cuidadores de pacientes en quimioterapia por Carey y cols. (12).

16. La Escala de Problemas del Cuidador de Kinney y Stephens, que mira a cuidadores de personas con demencia y es confiable para medir problemas de cuidado en la vida diaria (47).

17. La Escala de Problemas e Inspiraciones al Dar Cuidado, de Kinney y Stephens, que revisó la carga en cuidadores de personas con demencia para valorar elementos de la D CF-R como la dependencia de la persona con demencia y la satisfacción del cuidador (48).

18. La Escala de Frustración de Motenko, que revisó la DCF-R en cuidadoras de sus esposos con demencia en el hogar (49).

19. La Escala de Estrés del Cuidador, de Pearlin y cols., basada en el modelo de estrés de los cuidadores que estudió la D CF-R en cuidadores 
de personas con enfermedad de Alzheimer (50) y en cuidadores de personas con anorexia nerviosa (51).

20. La Escala de Carga del Cuidado Percibido por el Cuidador, de Stommel y cols. (52), con una versión adaptada al hindi por Gupta, para cuidadores asiático-americanos (53).

21. La Escala del Apoyo Social Percibido para el Cuidar, de Goodman, que miró cuidadores que atienden como miembros de un grupo que apoyó la DCF-R a través de actividades dirigidas al cuidador (54).

22. La Escala de Carga Percibida por el Cuidador, de Strawbridge y Wallhagen, para cuidadores de personas con ECNT (55).

23. El Monitoreo de la Carga del Cuidador, de Vitalino y cols., estudiada en esposas de pacientes con enfermedad de Alzheimer (56).

24. El Inventario de Dar Cuidado Familiar de Archbold y cols., en cuidadores que asisten a un anciano dependiente (57).

25. La Estimación del Impacto del Cuidado, de Orbell y cols., para cuidadores de personas ancianas (58).

26. La Valoración de la Reacción del Cuidador, de Given y cols., en cuidadores de ancianos enfermos (59).

27. La Escala de Carga del Cuidador, propuesta por Gerritsen y Van der Ende, desarrollada con cuidadores de pacientes psicogeriátricos (60).

28. La Escala de Autoeficacia en el Dar Cuidado, de Denney para cuidadores de personas con Alzheimer (61). Esta escala demostró ser útil en la validación de un modelo teórico para el cuidado de cuidadores de ancianos con demencia en Taiwán (62).

29. La Herramienta de Medición Carga y Bienestar Genérico del Cuidador, presentada por Stull y cols., para cuidadores de personas ancianas (63). Ha sido empleada en el contexto japonés (64).

30. El Cuestionario de Problemas de los Padres Cuidadores, de England y Roberts, utilizada con hijos adultos cuidadores de personas con trastornos neurológicos (65).

31. El Cuestionario del Sentido de Competencia, de Vernooij-Dassen y cols., desarrollada en hijos adultos cuidadores de personas con trastornos neurológicos (66). Tiene una versión corta desarrollada para la clínica (67). Al desarrollar nuevas pruebas psicométricas de la original se encontró que la única escala suficientemente válida al usar la herramienta en diferentes poblaciones de cuidadores es la de consecuencias de cuidar (68).

32. El Índice Multidimensional de Estrés del Cuidador, de Stull, (69), que hace hincapié en el tiempo de cuidado, cuenta con una versión adaptada para cuidadores de personas con Parkinson, propuesta por Factor (70).

33. La Escala de Carga del Cuidador, de Elmstahl y cols. Fue empleada en cuidadores de personas con secuelas de enfermedad cerebrovascular (71) y en cuidadores de pacientes sometidos a diálisis (72).

34. La Entrevista de la Actividad del Cuidador, de Davis y cols., para cuidadores de personas con enfermedad de Alzheimer. Esta permite predecir costo del cuidado a partir del tiempo dedicado y puede ser autoadministrado. Ha sido aplicado en díadas cuidador-persona con 
Alzheimer. La entrevista tiene debilidades psicométricas y solo recomienda el uso para profundizar aspectos de valoración clínica (73).

35. La Escala de Dar Cuidado Familiar de Schofield y cols., trabajada con cuidadores de ancianos de personas con ECNT y con dependencia por discapacidad de cualquier clase, se basa en la experiencia de ser cuidador de diferentes edades y empleada en la investigación y la clínica (74).

36. La Escala de Recompensas del Cuidador, de Picot y cols., desarrollada con cuidadoras mujeres afroamericanas, cuidadores y cuidadoras blancos (75).

37. La Escala de Distrés del Cuidador Inventario Neuropsiquiátrico, de Kaufer y cols. (76), que cuenta con versión reducida para la clínica Kaufer y cols. Fue gestada con cuidadores de personas con enfermedad de Alzheimer. La versión aplicada en clínica demostró ser útil con algunas limitaciones para personas con insuficiente ilustración. Se recomienda revisar para población con diversidad étnica y cultural (77).

38. La Escala de resultados de Bakas, de Bakas y Champion, desarrollada con cuidadores de personas con enfermedad cerebrovascular. Esta puede ser autoadministrada (78).

39. El Cuestionario Encontrando Significado a través del Cuidado, de Farran y cols., que miró la experiencia de los cuidadores de personas con demencia. También puede ser autoadministrada y aplicada en la clínica y la investigación (79).

40. La Escala Subjetiva de Carga, de Matsuda, que surge de investigación con cuidadores de ancianos con demencia. Puede, como las anteriores, ser autoadministrada (80).

41. La Herramienta Autoeficacia del Cuidador, de Zeiss y cols., que surge del trabajo con cuidadores de ancianos con discapacidad en quienes revisa la autoeficacia del cuidador en un grupo terapéutico de intervención psicoeducativa; es decir, valora a través del cuidador la aplicación de la teoría de autoeficacia a la situación de dar cuidado (81).

42. E1 Îndice de Carga de Dar Cuidado, de Miyashita y cols. (82), que estudió los cuidadores familiares de pacientes con enfermedades neurológicas degenerativas o cerebrovasculares. Esta fue contrastada con la escala de Zarit (83) y demostró ser válida y confiable.

El análisis de estos estudios de medición de la carga a través de uno de los cuidadores como integrantes de la D CF-R permite ver que se ha hecho hincapié en ocho aspectos que incluyen la salud, el bienestar psicológico, las finanzas y costos de la vida social, la relación con la persona enferma, la carga en el hogar o familia, los problemas del receptor, los ajustes familiares requeridos y los aspectos positivos del cuidado, aunque la división de las medidas no presenta estas categorías como mutuamente excluyentes. Es evidente que en este grupo de medidas el impacto de la carga se valora bajo principios sistémicos, es decir, a pesar de que se privilegia como informante al cuidador familiar, la otra parte de la DCF-R está presente en las mediciones, y en muchas de ellas incluso se considera al grupo familiar. 


\section{La D-CF-R desde la perspectiva de los receptores como parte del grupo familiar}

En la búsqueda de cuestionarios de medición de carga solo se encontró el estudio que dio origen a la Entrevista del Impacto Familiar, generada por Covinsky (84). En la entrevista se mira al paciente como integrante de la DCF-R, y a su familia, para valorar la experiencia de unos y otros cuando estuvieron hospitalizados, seriamente enfermos. Tomaron población recientemente dada de alta y pusieron el relieve en el costo del cuidado en el ámbito familiar.

\section{La D-CF-R propiamente dicha para comprender la carga del cuidado de la ECNT}

En los estudios que miden la carga del cuidado de la ECNT se encontraron tres que miden la D CF-R propiamente dicha a través de escalas, aunque solo uno de ellos se desarrolló para este propósito específico. Estos son:

1. La Entrevista de Carga de Zarit, aplicada a diadas compuestas por el cuidador y el anciano dependiente, por Montorio y cols., donde los cuidadores perciben de manera negativa la relación interpersonal y cuando sienten incompetencia, repercute en su labor, en especial para las actividades de la vida diaria (85).

2. La Escala de Valoración de la Reacción del Cuidador, aplicada a díadas cuidador-persona en quimioterapia ambulatoria por Given, quien señala que el impacto del cuidador es más por la sintomatología que por la limitación física. Los síntomas de depresión se correlacionan en la díada y el optimismo del cuidador es predictor de su salud mental y capacidad de reacción (86).

3. La Escala de Unidad Funcional para Cuidar al Paciente, de Fredman y Daly, que mira la diada cuidador-persona con ECNT (87). Esta escala demostró gran utilidad como instrumento para valorar la estabilidad y la fortaleza de las díadas y es una escala corta, fácil de administrar y medir, con buena confiabilidad y validez y aplicable en la clínica y la investigación.

\section{La carga en la D-CF-R desde un cuidador externo al grupo familiar}

Dentro de los estudios de medición de la carga que miran la DCF-R través de un cuidador externo, se encontró el Reporte de Escala para el Cuidador de una Persona con VIH en fase sida, de Ferrari y sus cols. (88), empleado en cuidadores trabajadores y voluntarios. En este se buscó revisar la carga del cuidado en quienes a través del apoyo a estos pacientes apoyan a la DCF-R en su rutina. 


\section{Conclusiones}

A través de la revisión y análisis desarrollados se encontró que las diferentes formas de abordar la DCF-R modifica de manera importante su valoración y medición. Así, se puede analizar la díada en su contexto familiar, desde sus integrantes, de forma mixta o desde un cuidador externo. La carga de la DCF-R se ha medido, especialmente, a partir de la mirada de los cuidadores familiares. Comprender la relación de la carga con el proceso de conformación de la DCF-R permite incluir aspectos relevantes y oportunos dentro de las propuestas para la disminución de la carga del cuidado de la ECNT que, a su vez, mejoren el bienestar de las DCF-R.

\section{Referencias}

1. Sadavoy J, Wesson V. Refining dementia intervention: the caregiverpatient dyad as the unit of care. CMAJ. 2012;2:5-10.

2. Checton MG, Magsamen-Conrad K, Venetis MK, Greene K. A dyadic approach applying a developmental-conceptual model to couples coping with chronic illness. Health Educ Behav. 2015;42(2):257-67.

3. Chaparro Díaz L. Trascender en un "vínculo especial" de cuidado: el paso de lo evidente a lo intangible. Bogotá: Universidad Nacional de Colombia; 2009.

4. Kotronoulas G, Wengström Y, Kearney N. Sleep and sleep-wake disturbances in care recipient-caregiver dyads in the context of a chronic illness: a critical review of the literature. J Pain Symptom Manag. 2013;45(3):579-94.

5. Chaparro Díaz L. O vínculo especial de cuidado: construção de uma teoria fundamentada. Av Enferm. 2010;28(2):123-33.

6. Chaparro L. Cómo se constituye el "vínculo especial" de cuidado entre la persona con enfermedad crónica y el cuidador familiar. Aquichan. 2011;11(1):7-22.

7. Dehn L, Korn-Merker E, Pfäfflin M, Ravens-Sieberer U, May T. The Impact on Family Scale: Psychometric analysis of long and short forms in parents of children with epilepsy. Epilepsy Behav. 2014;32:21-6.

8. Werner H, Latal B, Valsangiacomo Buechel E, Beck I, Landolt MA. The impact of an infant's severe congenital heart disease on the family: a prospective cohort study. Congenit Heart Dis. 2014;9(3):203-10.

9. Mansfield AK, Keitner GI, Dealy J. The family assessment device: An update. Family Process. 2014.

10. Staccini L, Tomba E, Grandi S, Keitner GI. The evaluation of family functioning by the family assessment device: A systematic review of studies in adult clinical populations. Family Process. 2014.

11. Poulshock SW, Deimling GT. Families caring for elders in residence: Issues in the measurement of burden. J Gerontol. 1984;39(2):230-9.

12. Greeff AP, Van der Walt K-J. Resilience in families with an autistic child. Educ Train Autism Dev Disabil. 2010:347-55.

13. Nabors LA, Kichler JC, Brassell A, Thakkar S, Bartz J, Pangallo J, et al. Factors related to caregiver state anxiety and coping with a child's chronic illness. Fam Syst Health. 2013;31(2):171. 
14. Hua J, Wu Z-C, Gu G, Meng W. [Assessment on the validity and reliability of Family Environment Scale on Motor Development for Urban Pre-school Children]. Zhonghua liu xing bing xue za zhi=Zhonghua liuxingbingxue zazhi. 2012;33(5):464-9.

15. Bass DM, Bowman K. The transition from caregiving to bereavement: The relationship of care-related strain and adjustment to death. Gerontologist. 1990;30(1):35-42.

16. Sanjo M, Morita T, Miyashita M, Shiozaki M, Sato K, Hirai K, et al. Caregiving Consequences Inventory: a measure for evaluating caregiving consequences from the bereaved family member's perspective. PsychoOncology. 2009;18(6):657-66.

17. Simmons LA. Self-perceived burden in cancer patients: validation of the Self-perceived Burden Scale. Cancer Nurs. 2007;30(5):405-11.

18. Kowal J, Wilson KG, McWilliams LA, Péloquin K, Duong D. Selfperceived burden in chronic pain: relevance, prevalence, and predictors. Pain. 2012;153(8):1735-41.

19. Cousineau N, McDowell I, Hotz S, Hébert P. Measuring chronic patients' feelings of being a burden to their caregivers: development and preliminary validation of a scale. Med Care. 2003;41(1):110-8.

20. Bachner YG, O'Rourke N, Ayalon L, Bédard M. Comparison of caregiver responses to English and Hebrew language versions of an abridged Zarit Burden Interview. Aging Ment Health. 2011;15(3):370-5.

21. Brink P, Stones M, Smith TF. Confirmatory factor analysis of the burden interview of the caregivers of terminally ill home care clients. J Palliat Med. 2012;15(9):967-70.

22. Chattat R, Cortesi V, Izzicupo F, Del Re ML, Sgarbi C, Fabbo A, et al. The Italian version of the Zarit Burden Interview: a validation study. Int Psychogeriatr. 2011;23(05):797-805.

23. Merino-Soto C, Angulo-Ramos M. Validación en Chile de la escala de sobrecarga del cuidador de Zarit en sus versiones original y abreviada: corrección. Rev Méd Chile. 2013;141(8):1083-4.

24. Folkman S, Lazarus RS. An analysis of coping in a middle-aged community sample. J Health Soc Beh. 1980:219-39.

25. Vitaliano PP, Russo J, Carr JE, Maiuro RD, Becker J. The ways of coping checklist: Revision and psychometric properties. Multivariate Behav Res. 1985;20(1):3-26.

26. Menezes AR, Lavie CJ, DeSchutter A, Milani RV. Gender, race and cardiac rehabilitation in the United States: Is there a difference in care? Am J Med Sci. 2014;348(2):146-52.

27. Boots L, Vugt M, Knippenberg R, Kempen G, Verhey F. A systematic review of Internet based supportive interventions for caregivers of patients with dementia. Int J Geriatr Psychiatr. 2014;29(4):331-44.

28. Nogales-González C, Losada A, Romero-Moreno R. Confirmatory factor analysis of the Spanish version of the revised memory and behavior problems checklist. Int Psychogeriatr. 2014:1-10. 
29. Wang Q, Jiang F, Chen S, Tang S. Memory and behavior-related problems of patients with neurocognitive disorders and the attitudes of their caregivers. Family Medicine and Community Health. 2013;1(4):23-30.

30. Teri L, Truax P, Logsdon R, Uomoto J, Zarit S, Vitaliano PP. Assessment of behavioral problems in dementia: the revised memory and behavior problems checklist. Psychol Aging. 1992;7(4):622.

31. Cohen S, Kamarck T, Mermelstein R. A global measure of perceived stress. J Health Soc Behav. 1983:385-96.

32. Lee E-H. Review of the psychometric evidence of the perceived stress scale. Asian Nurs Res. 2012;6(4):121-7.

33. Kruithof WJ, Post MW, Visser-Meily JM. Measuring negative and positive caregiving experiences: A psychometric analysis of the Caregiver Strain Index Expanded. Clin Rehabil. 2015: feb 4.

34. Montgomery RJ, Gonyea JG, Hooyman NR. Caregiving and the experience of subjective and objective burden. Fam Relat. 1985:19-26.

35. Kosberg JI, Cairl RE. The cost of care index: A case management tool for screening informal care providers. Gerontologist. 1986;26(3):273-8.

36. Derogatis L. The Derogatis Stress Profile (DSP): quantification of psychological stress. Adv Psychosom Med. 1986;17:30-54.

37. Hansell P, Hughes C, Caliandro G, Russo P, Budin W, Hartman B, et al. Stress and social support in older caregivers of children with HIV/ AIDS: An intervention model. En: Invisible caregivers: Older adults raising children in the wake of HIV/AIDS. New York: Columbia University Press; 2002. p. 113-30.

38. Wells Y, Jorm A. Evaluation of a special nursing home unit for dementia sufferers: a randomised controlled comparison with community care. Aust N Z J Psychiatry. 1987;21(4):524-31.

39. Bindoff HP, Clifford CA, Young JL. Caregivers of family members with dementia and disability: A comparative study of wellbeing. J Fam Stud. 1997;3(2):183-95.

40. Novak M, Guest C. Application of a multidimensional caregiver burden inventory. Gerontologist. 1989;29(6):798-803.

41. Caserta MS, Lund DA, Wright SD. Exploring the Caregiver Burden Inventory (CBI): further evidence for a multidimensional view of burden. Int J Aging Hum Dev. 1996;43(1):21-34.

42. Chou K-R, Jiann-Chyun L, Chu $\mathrm{H}$. The reliability and validity of the Chinese version of the caregiver burden inventory. Nurs Res. 2002;51(5):324-31.

43. Lawton MP, Kleban MH, Moss M, Rovine M, Glicksman A. Measuring caregiving appraisal. J Gerontol. 1989;44(3):P61-P71.

44. Hughes CB, Caliandro G. Effects of social support, stress, and level of illness on caregiving of children with AIDS. J Pediatr Nurs. 1996;11(6):347-58.

45. Sevick MA, Sereika S, Hoffman LA, Matthews JT, Chen GJ. A confirmatory factor analysis of the caregiving appraisal scale for 
caregivers of home-based ventilator-assisted individuals. Heart \& Lung. 1997;26(6):430-8.

46. Struchen MA, Atchison TB, Roebuck TM, Caroselli JS, Sander AM. A multidimensional measure of caregiving appraisal: validation of the Caregiver Appraisal Scale in traumatic brain injury. J Head Trauma Rehabil. 2002;17(2):132-54.

47. Oberst MT, Thomas SE, Gass KA, Ward SE. Caregiving demands and appraisal of stress among family caregivers. Cancer Nurs. 1989;12(4):209-15.

48. Carey PJ, Oberst MT, McCubbin MA, Hughes SH, editors. Appraisal and caregiving burden in family members caring for patients receiving chemotherapy. Oncol Nurs Forum; 1990 Nov-Dec;18(8):1341-8.

49. Kinney JM, Stephens MAP. Caregiving Hassles Scale: Assessing the daily hassles of caring for a family member with dementia. Gerontologist. 1989;29(3):328-32.

50. Motenko AK. The frustrations, gratifications, and well-being of dementia caregivers. Gerontologist. 1989;29(2):166-72.

51. Pearlin LI, Mullan JT, Semple SJ, Skaff MM. Caregiving and the stress process: An overview of concepts and their measures. Gerontologist. 1990;30(5):583-94.

52. Kyriacou O, Treasure J, Schmidt U. Understanding how parents cope with living with someone with anorexia nervosa: Modelling the factors that are associated with carer distress. Int J Eat Disord. 2008;41(3):233-42.

53. Stommel M, Given CW, Given B. Depression as an overriding variable explaining caregiver burdens. J Aging Health. 1990;2(1):81-102.

54. Gupta R. The revised caregiver burden scale: A preliminary evaluation. Res Social Work Prac. 1999;9(4):508-20.

55. Goodman CC. Perceived social support for caregiving: Measuring the benefit of self-help/support group participation. J Gerontol Social Work. 1991.

56. Strawbridge WJ, Wallhagen MI. Impact of family conflict on adult child caregivers. Gerontologist. 1991;31(6):770-7.

57. Vitaliano PP, Russo J, Young HM, Becker J, Maiuro RD. The screen for caregiver burden. Gerontologist. 1991;31(1):76-83.

58. Archbold P, Stewart B, Greenlick M, Harvath T. The clinical assessment of mutuality and preparedness in family caregivers to frail older people. En: Key aspects of elder care: Managing falls, incontinence, and cognitive impairment. New York: Springer; 1992. p. 328-39.

59. Orbell S, Hopkins N, Gillies B. Measuring the impact of informal caring. J Community Appl Soc. 1993;3(2):149-63.

60. Given CW, Given B, Stommel M, Collins C, King S, Franklin S. The caregiver reaction assessment (CRA) for caregivers to persons with chronic physical and mental impairments. Res Nurs Health. 1992;15(4):271-83.

61. Gerritsen J, Van der Ende P. The development of a care-giving burden scale. Age Ageing. 1994;23(6):483-91. 
62. Denney JE. Development and validation of the caregiving self-efficacy scale. New York: University of Missouri-Columbia; 1992.

63. Stull DE, Kosloski K, Kercher K. Caregiver burden and generic wellbeing: opposite sides of the same coin? Gerontologist. 1994;34(1):88-94.

64. Sugihara Y, Sugisawa H, Nakatani Y, Hougham GW. Longitudinal changes in the well-being of Japanese caregivers: Variations across kin relationships. J Gerontol B Psychol Sci Soc Sci. 2004;59(4):P177-P84.

65. England M, Roberts BL. Theoretical and psychometric analysis of caregiver strain. Res Nurs Health. 1996;19(6):499-510.

66. Vernooij-Dassen MJ, Persoon JM, Felling AJ. Predictors of sense of competence in caregivers of demented persons. Soc Sci Med. 1996;43(1):41-9.

67. Vernooij-Dassen M, Felling A, Brummelkamp E, Dauzenberg M, Van Den Bos G, Grol R. Assessment of caregiver's competence in dealing with the burden of caregiving for a dementia patient: a Short Sense of Competence Questionnaire (SSCQ) suitable for clinical practice. J Am Geriatr Soc. 1999;47(2):256-7.

68. Jansen AP, van Hout HP, van Marwijk HW, Nijpels G, Gundy C, VernooijDassen MJ, et al. Sense of competence questionnaire among informal caregivers of older adults with dementia symptoms: a psychometric evaluation. Clin Pract Epidemiol Ment Health. 2007;3(1):11.

69. Stull D. The Multidimensional Caregiver Strain Index (MCSI). Its measurement and structure. J Clin Geropsychol. 1996;2(3):175-96.

70. Factor S, Weiner W, Factor S, Weiner W. Parkinson's disease: diagnosis \& clinical management. New York: Demos Medical Publishing; 2007.

71. Elmståhl S, Malmberg B, Annerstedt L. Caregiver's burden of patients 3 years after stroke assessed by a novel caregiver burden scale. Arch Phys Med Rehabil. 1996;77(2):177-82.

72. Belasco A, Barbosa D, Bettencourt AR, Diccini S, Sesso R. Quality of life of family caregivers of elderly patients on hemodialysis and peritoneal dialysis. American Journal of Kidney Diseases. 2006;48(6):955-63.

73. Davis KL, Marin DB, Kane R, Patrick D, Peskind ER, Raskind MA, et al. The Caregiver Activity Survey (CAS): development and validation of a new measure for caregivers of persons with Alzheimer's disease. Int J Geriatr Psychiatry. 1997;12(10):978-88.

74. Schofield HL, Murphy B, Herrman H, Bloch S, Singh B. Family caregiving: measurement of emotional well-being and various aspects of the caregiving role. Psychol Med. 1997;27(03):647-57.

75. Fulton Picot SJ, Youngblut J, Zeller R. Development and testing of a measure of perceived caregiver rewards in adults. J Nurs Meas. 1997;5(1):33-52.

76. Kaufer DI, Cummings JL, Christine D, Bray T, Castellon S, Masterman $\mathrm{D}$, et al. Assessing the impact of neuropsychiatric symptoms in Alzheimer's disease: the Neuropsychiatric Inventory Caregiver Distress Scale. J Am Geriatr Soc. 1998 Feb;46(2):210-5. 
77. Kaufer DI, Cummings JL, Ketchel P, Smith V, MacMillan A, Shelley T, et al. Validation of the NPI-Q, a brief clinical form of the Neuropsychiatric Inventory. The J Neuropsychiatry Clin Neurosci. 2000;12(2):233-9.

78. Bakas T, Champion V. Development and psychometric testing of the Bakas Caregiving Outcomes Scale. Nurs Res. 1999;48(5):250-9.

79. Farran CJ, Miller BH, Kaufman JE, Donner E, Fogg L. Finding meaning through caregiving: Development of an instrument for family caregivers of persons with Alzheimer's disease. J Clin Psychol. 1999;55(9):1107-25.

80. Matsuda O. Reliability and validity of the subjective burden scale in family caregivers of elderly relatives with dementia. Int Psychogeriatr. 1999;11(2):159-70.

81. Zeiss AM, Gallagher-Thompson D, Lovett S, Rose J, McKibbin C. Selfefficacy as a mediator of caregiver coping: Development and testing of an assessment model. J Clin Geropsychol. 1999;5(3):221-30.

82. Miyashita M, Yamaguchi A, Kayama M, Narita Y, Kawada N, Akiyama $\mathrm{M}$, et al. Validation of the Burden Index of Caregivers (BIC), a multidimensional short care burden scale from Japan. Health Qual Life Outcomes. 2006;4(1):52.

83. McPherson CJ, Wilson KG, Brajtman S, Lobchuk M. Self-perceived burden to others: patient and family caregiver correlates. J Palliat Care. 2007;23(3):135.

84. Covinsky KE, Goldman L, Cook EF, Oye R, Desbiens N, Reding D, et al. The impact of serious illness on patients' families. JAMA. 1994;272(23):1839-44.

85. Montorio Cerrato I, Fernández de Trocóniz MI, López López A, Sánchez Colodrón M. La entrevista de carga del cuidador: utilidad y validez del concepto de carga. Anales de Psicología. 1998;14(2):229-48.

86. Given CW, Stommel M, Given B, Osuch J, Kurtz ME, Kurtz J. The influence of cancer patients' symptoms and functional states on patients' depression and family caregivers' reaction and depression. Health Psychol. 1993;12(4):277.

87. Fredman L, Daly M. Patient-caregiver functional unit scale: a new scale to assess the patient-caregiver dyad. Fam Med. 1997;29(9):658-65.

88. Ferrari JR, Mccown W, Pantano J. Experiencing Satisfaction and Stress as an Aids Care Provider The AIDS Caregiver Scale. Eval Health Prof. 1993;16(3):295-310. 